East African Medical Journal Vol. 85 No. 11 November 2008

COMPARISON OF BLOOD SMEAR MICROSCOPY TO A RAPID DIAGNOSTIC TEST FOR IN-VITRO TESTING FOR P. FALCIPARUM MALARIA IN KENYAN SCHOOL CHILDREN

C.G. Neumann, MD., MPH., Professor, Departments of Paediatrics and Community Health Sciences, Schools of Public Health and Medicine, University of California, Los Angeles, Los Angeles, CA, United States, 90095, N.O. Bwibo, MBBS, MPH, Professor Emeritus, Department of Paediatrics and Child Health, College of Health Sciences, University of Nairobi, P. O. Box 19676-00202, Nairobi, Kenya, J.H. Siekmann, PhD, Department of Nutritional Sciences, University of California, Davis, Davis, CA, United States, 95616. Currently (2007) Technical Officer, Department of Nutrition for Health and Development at WHO, Geneva E.D. McLean, PhD, Department of Nutritional Sciences, University of California, Davis, Davis, CA, United States, 95616. Currently (2006) Technical Officer for the Micronutrient Program in the Department of Nutrition for Health and Development at WHO, Geneva, B. Browdy, PhD, Statistician, UCLA School of Public Health, Los Angeles, CA, United 2 States, 90095. Currently emeritus. N. Drorbaugh, MA, MPH, Staff Research Associate II, UCLA School of Public Health, Los Angeles, CA, United States, 90095.

Request for reprints to: Dr. C.G. Neumann, UCLA School of Public Health, P.O. Box 951772, Los Angeles, CA 90095-1772

\title{
COMPARISON OF BLOOD SMEAR MICROSCOPY TO A RAPID DIAGNOSTIC TEST FOR IN-VITRO TESTING FOR P. FALCIPARUM MALARIA IN KENYAN SCHOOL CHILDREN
}

\author{
C.G. NEUMANN, N.O. BWIBO, J.H. SIEKMANN, E.D. McLEAN, \\ B. BROWDY and N. DRORBAUGH
}

\begin{abstract}
Objective: To compare the diagnostic performance of microscopy using Giemsastained thick and thin blood smears to a rapid malaria dipstick test (RDT) in detecting $P$. falciparum malaria in Kenyan school children.

Design: Randomised, controlled feeding intervention trial from 1998-2001.

Setting: Rural Embu district, Kenya. The area is considered endemic for malaria, with four rainy seasons per year. Chloroquine resistance was estimated in $80 \%$ of patients. Children had a spleen rate of $45 \%$.

Subjects: A sample of 515 rural Kenyan primary school children, aged 7-11 years, who were enrolled in a feeding intervention trial from 1998-2001.

Main outcome measures: Percent positive and negative P. falciparum malaria status, sensitivity, specificity and positive and negative predictive values of RDT.

Results: For both years, the RDT yielded positive results of $30 \%$ in children compared to microscopy (17\%). With microscopy as the "gold standard," RDT yielded a sensitivity of $81.3 \%$ in 1998 and $79.3 \%$ in 2000 . Specificity was $81.6 \%$ in 1998 and $78.3 \%$ in 2000 . Positive predictive value was $47.3 \%$ in 1998 and $42.6 \%$ in 2000, and negative predictive value was $95.6 \%$ in 1998 and $94.9 \%$ in 2000 .

Conclusion: Rapid diagnostic testing is a valuable tool for diagnosis and can shorten the interval for starting treatment, particularly where microscopy may not be feasible due to resource and distance limitations.
\end{abstract}

\section{INTRODUCTION}

Malaria is among the leading causes of childhood morbidity and mortality, particularly in sub-Saharan Africa and Southeast Asia (1). In many areas, death rates from malaria exceed those from HIV / AIDS and other infections, with $P$. falciparum responsible for the greatest number of deaths. Multiple factors contribute to the prevalence and severity of malaria.
Theseare lack of preventive and protective measures such as chemical and environmental control of vector populations and increasing mosquito and parasite resistance to insecticides and drugs, respectively $(2,3)$. Problems with distribution and underutilisation of insecticide impregnated bed nets limit their use even though they have been demonstrated to be effective in reducing malaria incidence $(4,5)$. 
Moreover, access to diagnostic testing and effective antimalarial drugs is limited. Poor access to health facilities is a major barrier to early malaria diagnosis and treatment. Moreover, microscopy may not be available in many rural areas where trained personnel and adequate laboratory facilities are unavailable. Despiteefforts made to decrease the cost of effective antimalarial drugs for consumers, competing financial demands may make it difficult for consumers to purchase adequate amounts of these (6). In 2000, in the 42 countries with $90 \%$ of worldwide childhood mortality, only $29 \%$ (ranging from 3-66\%) of children under age five years had access to antimalarial drugs (7). Poverty creates an inability to pay for clinic visits, malaria testing, and purchase of drugs (8). Home treatmentmay beinadequate, withincorrectdrugdoses and / or expired drugs. Early diagnosis and treatment are of utmost importance to reduce morbidity and mortality from malaria, particularly from $P$. falciparum malaria. Early diagnosis facilitates early initiation of appropriate treatment, which could prevent severe complications and mortality. In malarious areas of Africa, fevers are often assumed to be malarial, and overdiagnosis of malaria may lead people to purchase and utilise malarial medications for non-malarial fevers. Unnecessary treatment with ineffective drugs and dosages contributes to development of parasite resistance.

Microscopy of blood smears stained using either Giemsa or Field-stains is universally considered the standard for malaria diagnosis (9). Yet, errors in diagnostic accuracy are an inherent limitation of microscopy (10), as it requires properly prepared and stained smears, well-trained microscopists, appropriate laboratory equipment, adequate light source, and periodic duplicate readings by a second microscopist for quality control-resources which are unavailable in many malaria endemic rural areas. Diagnosis of malaria could be improved and facilitated through rapid diagnosis. Aside from rapid dipstick technology, various techniques have been explored including the Quantitative Buffy Coat (QBC) method. While the QBC has been shown to have high sensitivity and specificity, it requires a centrifuge to prepare either capillary or venous blood samples for examination (11).

Rapid diagnostictests (RDT) using dipsticks have been developed to diagnose the main types of malaria and do not require use of a laboratory. These are availablefor single species or all four species of malaria. These RDT have been developed and tested in nonimmune travellers, clinical cases, and communities in endemic areas. A RDT detecting plasmodial histidine-rich protein 2 (HRP-2) showed a high level of sensitivity and specificity in non-immune travellers (sensitivity of $92.5 \%$ and specificity of 98.3\%) (12). Another study using an HRP-2 dipstick in infected travellers found sensitivity of $90 \%$ and specificity of $96 \%$ (13). High sensitivity (95.4\%) and specificity (94.1\%) was found using RDT in children admitted to an outpatient hospital with fever or history of fever in Papua, New Guinea (14). The study reported here sought to compare the diagnostic performance of microscopy using Giemsa-stained thick and thin blood smears to RDT in detecting P. falciparum malaria in Kenyan primary school children who were participating in a feeding intervention study.

\section{MATERIALS AND METHODS}

Sample and study area: A sample of 515 rural Kenyan primary school children, aged 7-11 years, enrolled in a randomised, controlled feeding intervention trial from 1998-2001 was utilised. These children lived in rural Embu district southeast of Mt. Kenya (1200$2500 \mathrm{~m}$ ), usually with two long and two short rainy seasons per year. The area is endemic for P. falciparum malaria, and the health centre and district hospital estimated that chloroquine resistance was present in approximately $80 \%$ of patients. The reported prevalence of malaria by parents in the study children at baseline was $17 \%$. Other baseline characteristics of children are summarised in Table 1.

Table 1

Baseline characteristics of children

\begin{tabular}{lc} 
Age & $7.4 \pm 1.2$ \\
Male $(\%)$ & 52 \\
Infection* $(\%)$, examined in 523 & 17.8 \\
Enlarged spleen (\%), examined in 398 & 45.4 \\
Faecal parasites, examined in 510 children \\
Entamoeba histolytica (\%) \\
Giardia lamblia (\%) & 17.6 \\
Hookworm (\%) & 16 \\
Ascaris lumbricoides (\%) & 0.9 \\
Escherichia coli (\%) & 4.7 \\
\hline
\end{tabular}

${ }^{*}$ C-reactive protein $>10 \mathrm{mg} / \mathrm{L}$

Design: The 515 children enrolled in the feeding intervention were tested for malaria using both microscopy and RDT at baseline, one year, and two years after the feeding intervention began. Ongoing morbidity data was collected at one to two month intervals by interviewing mothersusing aquestionnaire and by physical inspection of the child conducted by enumerators trained by nurses and doctors to identify signs and symptoms of illness including fever, chills, malaria and other morbidity.

Methods for malaria detection: Three methods detailed below were used to assess malaria status: i) microscopy; ii) RDT strips, a rapid blood test; and iii) 
a monthly morbidity determination based on reported and observed signs and symptoms such as fever, chills, and caretaker's clinical diagnosis of malaria. Blood samples were obtained by venipuncture or finger stick annually between 1998 and 2000.

Microscopy was performed on thick and thin blood smears, stained with Giemsa and allowed to dry. Stained slides were read independently by two very experienced senior technicians from the University of Nairobi, Department of Medical Microbiology in 1998 and 1999 for quality control purposes. In 2000, one microscopist was used, with periodic quality control checks by a second technician. Malaria parasites (mps) per 100 red blood cells were counted in the thin smear, and mps per 200 white blood cells were counted in the thick smear. The technicians' independent readings demonstrated a high percentage of agreement (kappa $=0.9039$ ). Microscopy data from 1999 was excluded because the slides were damaged.

Rapid testing for malaria via dipstick antigen capture was performed using the Vision Biotech Pf malaria test (Cape Town, South Africa), a rapid qualitative method that detects $P$. falciparum in either anti-coagulated venous blood, capillary blood, or serum using an immunochromatographic strip. The Vision Biotech test is an antigen-capture assay which detects the presence of specific soluble histidinerich protein 2 (HRP-2) present in and released from infected and lysed RBCs. A capture monoclonal antibody is immobilised on the test strip, which binds to the histidine rich protein. The reagent, coated with specific antibodies, binds to the antigen-antibody complex producing a red line on the strip. A control (C) strip and the test strip ( $\mathrm{T}$ ) are assessed to determine the presence or absence of P.falciparum malaria. Blood or serum was drawn up in a capillary tube (either $5 \mu \mathrm{L}$ or $10 \mu \mathrm{L}$ may be used) and was then transferred to the test strip by gently touching the nozzle to the well (Figure 1a). Four drops of reaction buffer were then placed into the well (Figure 1b). After exactly 15 minutes the results were obtained by comparing the $\mathrm{C}$ line to the $\mathrm{T}$ line (Figure 1c). Two lines signified the presence of $P$. falciparum antigens. A control line alone indicated that the test was performed correctly but no antigens were present. No visible lines indicated an invalid test result.

Figure 1

Rapid diagnostic malarial test for P. falciparum

a) Blood is transferred to the well

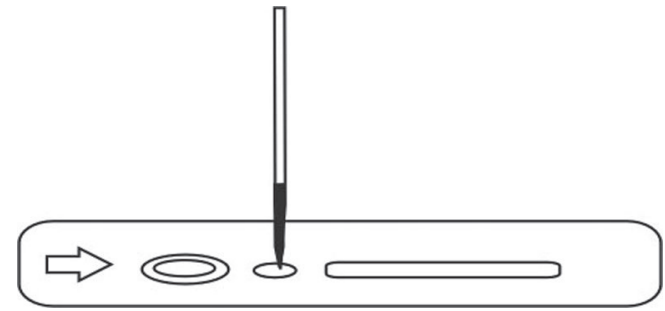

b) Four drops of reaction buffer are added to the well

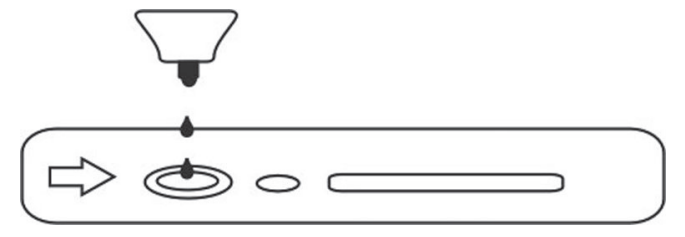

c) Results of the rapid test are indicated on the test strip



Morbidity data was obtained by home visits conducted every two months using a questionnaire administered to the child's caretaker inquiring about signs and symptoms of illness on the day of the visit and the preceding six days. The questionnaire initially consisted of open-ended questions asking caretakers to describe any illness, followed by a structured questionnaire with probing questions about signs and symptoms which comprised a diagnosis or illness categories (15). Fever, chills, and the use of the term malaria were specifically asked about. If the child was at home because of illness, oral temperature was taken and observations of the sick child were recorded following a strict protocol. Morbidity results only from July 2000 home visit are reported in this paper as this was the only year with blood testing and microscopy data to correspond with morbidity data. The July home visit was the visit which most closely corresponded to the date of the annual malaria blood test. Morbidity data was not available for 1998.

Statistical analyses: Sensitivity and specificity were calculated using results from RDT and microscopy. Sensitivity and specificity were calculated using data from 1998 and 2000. In calculations of sensitivity and specificity, data were excluded for children who lacked both microscopic results and for children who lacked a RDT strip result. If children had at least one microscopic result and a RDT result for the same blood sample, their data was included for analysis. Sensitivity for RDT was calculated as children 
positive by both methods divided by (positives by both methods + positives by RDT alone); specificity as children negative by both methods divided by (negatives by both methods + positives by RDT alone). Positive predictive value was calculated as positives by both methods divided by the total number who tested positive, and negative predictive value was calculated as children negative by both methods divided by the total number who tested negative. Approval by the UCLA Human Subject Protection Committee, the Ethics Committee of the University of Nairobi School of Medicine, and the Office of the President were obtained prior to commencing the study. Verbal informed consent by parents and assent by children and community permission were also obtained.

\section{RESULTS}

Overall, the three types of diagnostic methods produced widely differing results. Rapid diagnostic tests yielded the highest rates of positives, detecting nearly double the number of children with $P$. falciparum as microscopy and two to three times greater the number diagnosed with "clinical malaria" by morbidity reports (Table 2). Using RDT results of those who were tested each year, $6 \%$ of the sample tested positive both years and $39 \%$ tested negative. Morbidity reports for 2000 indicated that 458 (7.0\%) children experienced fever and chills and were identified as having malaria by the caretaker.

\section{Table 2}

Descriptive results for RDT, microscopy, and reported morbidity data

\begin{tabular}{lcccc}
\hline & \multicolumn{4}{c}{ Positive } \\
Year & \multicolumn{3}{c}{1998} & 2000 \\
& No. & $(\%)$ & No. & $(\%)$ \\
\hline & & & & \\
RDT & 515 & 31.7 & 346 & 31.2 \\
Microscopy & 456 & 16.9 & 360 & 17.8 \\
Reported and & & & & \\
observed morbidity* & - & & 458 & 7.0 \\
\hline
\end{tabular}

* Fever, chills, and presumptive diagnosis of malaria by caretaker

- Morbidity data temporally corresponding toblood tests not available for 1998

Using microscopy as the "gold standard," the RDT yielded a sensitivity of $81.3 \%$ in 1998 and a sensitivity of $79.3 \%$ in 2000 and specificity of $81.6 \%$ in 1998 and 78.3\% in 2000 (Table 3). Using data from 1998, a positive predictive value of $47.3 \%$ was obtained, while in 2000 the positive predictive value was $42.6 \%$. The calculated negative predictive value was $95.6 \%$ in 1998 and $94.9 \%$ in 2000. Data from 445 children were included in analysis for 1998 and 344 children in 2000. Utilising microscopy as the "gold standard", the RDT has a low rate of false negatives (about 3\%) but a higher rate of false positives (15-28\%) (Table 4).

Table 3

Results by year (RDT compared to microscopy) in percentage

\begin{tabular}{lcc}
\hline & $\begin{array}{c}1998 \\
(\mathrm{n}=445)\end{array}$ & $\begin{array}{c}2000 \\
(\mathrm{n}=344)\end{array}$ \\
\hline Sensitivity & 81.3 & 79.3 \\
Specificity & 81.6 & 78.3 \\
Positive predictive value & 47.3 & 42.6 \\
Negative predictive value & 95.6 & 94.9 \\
\hline
\end{tabular}

Table 4

Percent agreement, false negatives, and false positives (RDT compared to microscopy) by year

\begin{tabular}{lrr}
\hline & 1998 & 2000 \\
\hline Agreement & 81.6 & 78.5 \\
False negatives & 3.1 & 3.5 \\
False positives & 15.3 & 18.0 \\
\hline
\end{tabular}

\section{DISCUSSION}

The use of a RDT for P. falciparum malaria detection in a group of children living in an endemic area yielded nearly double the positive results than microscopy using Giemsa-stained blood smears. Previous studies of asymptomatic individuals comparing microscopy to RDT that screen for HRP-2 in endemic areas yielded average sensitivity of $88 \%$ (range $86-90 \%$ ) (10). The sensitivity values obtained in our study are somewhat lower than these results, sensitivity of $(81.33 \%$ in 1998 and $79.3 \%$ in 2000). In terms of specificity, our obtained specificity values $(81.6 \%$ in 1998 and $78.3 \%$ in 2000 ) are lower than in field studies of asymptomatic individuals in endemic areas $(99.90 \%$, range 99.7 $100 \%$ ) (10). However, one study of a RDT detecting HRP-2 antigen among Kenyans in an endemic area yielded a specificity of $88 \%(84-92 \%, n=285)$, close to that obtained in the present study (16). In the epidemiologic sense, assessing specificity of RDT in endemic areas is difficult. There is no definitive way 
of knowing that a negative microscopy test result coincides with absence of malaria infection since commonly used microscopy does not perform as a true "gold standard" (16).

The lower specificity obtained in our study may also be due in part to the population sampled in our study, which included both symptomatic and asymptomatic children. Some children who had experienced fever and chills were reported by caretakers as having clinical malaria. Likewise, the low positive predictive value of RDT found in this population could be related to the fact that tests were performed in asymptomatic and symptomatic children. Prevalence of a condition in the population tested influences the positive predictive value. So, had tests been carried only in symptomatic children, the positive predictive value obtained would have been higher. The RDT yielded a high negative predictive value (95.6\% in 1998 and $94.9 \%$ in 2000). Hence, if children tested negative for malaria by the RDT, they were likely to also be negative for malaria by microscopy.

Nearly double the number of positive findings for malaria was detected by RDT than by microscopy, in $31 \%$ of children versus $17 \%$, respectively. Concern has been expressed about false positives using HRP2 dipstick tests due to persistent HRP-2 antigen in circulation for up to two weeks after viable parasites are eliminated from blood. Presence of gametocytes has also been suggested as a cause of false positive results, but Beadleet al (16) analysis of gametocytaemia among subjects with false-positive dipsticks found no indication that gametocy te presence was responsible for false positive results.

Clinical diagnosis of malaria is problematic as many of the signs and symptoms are very nonspecific. Morbidity reports are a poor method to detect malaria. The figures obtained were much lower than the results obtained for RDT and microscopy. Mild cases of malaria are likely under-diagnosed by caretakers. On the other hand, clinicalmalaria is often overdiagnosed as any febrile event tends to be identified as malaria by the mother or caretaker. Also the timing of the blood testing for malaria and the morbidity home visits were not temporally coordinated (blood test once a year and home visits every two months). Although we picked the home visit for morbidity as close to the blood test as possible, these often did not coincide exactly. The reported symptoms are problematic because the July home visit did not take place on the same day, so reported symptoms do not exactly coincide with the blood testing as laboratory tests took place throughout the months of July and August. Additionally, the July home visit did not coincide with the season of rainfall. The heaviest rains occur in April and November, so in July there were still some residual swamps from the spring rains.
A RDT allows for rapid diagnosis of malaria as it only requires 15-20 minutes to obtain results and can be carried out by a trained community health worker (CHW) close to a person's home without travel by foot, often 3-5 km, to a health centre. No laboratory, electricity, or microscope is required. Rapid diagnostic testing has the potential to provide much earlier diagnosis than microscopy at a health facility and could allow the CHW to initiate appropriate treatment immediately after diagnosis is made. In contrast, a visit to a health centre may not be feasible given the distance and the prohibitive cost of the visit, the microscopic examination of blood, and any recommended medications may result in delays in seeking medical care or not seeking medical care altogether.

Use of RDT by trained volunteer barangay (village) health workers in the rural Philippines was well accepted and accurately performed, resulting in higher sensitivity than symptoms or microscopic examination of slides prepared by local health workers (17). In addition, patients expressed a preference for the RDT, and increases were seen in compliance and treatment-seeking behaviour (17). Rapid diagnostic examshavealso been effective, allowing for immediate administration of treatment in peripheral areas where access to microscopy diagnosis and health centres is difficult in Brazil and Thailand $(18,19)$.

While use of a RDT would not address the expense of malarial medications, it represents a feasible and relatively inexpensive diagnostic tool for malaria with potential to reduce mortality and morbidity from malaria through early diagnosis and treatment. The cost of RDT test strips detecting only $P$. falciparum is under US\$1.00, while test strips detecting multiple strains cost somewhat more than US\$1.00. This cost is relatively low compared to the cost of maintaining a laboratory technician, a microscope, a light source, slides, and stains. Rapid diagnostic tests are now available from many biotechnology companies in the United States, Europe, Africa, and India. In addition, RDT technology has potential to reduce inadequate home treatment with inadequate doses and inappropriate antimalarial drugs in the market by accurately diagnosing malaria.

Despite these advantages, RDT does not allow examination of parasite loads; these would have to be assessed via microscopy. The RDT used in this study only tests for P. falciparum; however, multivalent RDT are now available and under further development to allow for diagnosis of all four species of malaria parasites. Maintaining a steady supply of RDT may be problematic given logistic and distribution limitations in certain areas. Adequate distribution and storage networks and facilities are necessary to ensure that tests provided arenot damaged by heat and humidity 
(20). Health policy, administrative capacities, and knowledge of the status of malaria drug resistance are needed to address problems with access to effective malarial medication following diagnosis and distribution of medications. Rapid diagnostic tests should be integrated with the evaluation of other febrile illnesses, particularly in high risk groups such as young children and pregnant women (20). Further studies are badly needed comparing the actual time to diagnosis and treatment of malaria and relative cost and health outcomes with the use of RDT by CHWs with prompt diagnosis and appropriate treatment.

\section{ACKNOWLEDGEMENTS}

To Mr. J. Mudasia and Mr. P. Mulega Chitayi both laboratory technicians from the Department of Medical Microbiology, University of Nairobi.

\section{REFERENCES}

1. World Health Organisation. World Health Report: Shaping the Future. Geneva: World Health Organisation; 2003.

2. Brooke, B.D., Hunt, R.H., Chandre, F. et al. Stable chromosomal inversion polymorphisms and insecticide resistance in the malaria vector mosquito Anopheles gambiae (Diptera: Culicidae). J. Med. Entomol. 2002; 39: 568-573.

3. Olliaro, P.Drug resistance hampers our capacity to roll back malaria. Clin. Infect. Dis. 2005; 41: S247-S257.

4. Nevill, C.G., Some, E.S., Mung'ala, V. O. et al. Insecticide-treated bed nets reduce mortality and severe morbidity from malaria among children on the Kenyan coast. Trop. Med. Int. Health. 1996; 1: 139-146.

5. ter Kuile, F.O., Terlouw, D.J., Phillips-Howard, P.A. et al. Impact of permethrin-treated bed nets on malaria and all-cause morbidity in young children in an area of intense perennial malaria transmission in western Kenya: cross-sectional survey. Am. J. Trop. Med. Hyg. 2003; 68:100-107.

6. Global Partnership to Roll Back Malaria. Improving Access to Antimalarial Medicines. Geneva: World Health Organisation Malaria Control Department; 2003

7. UNICEF. The State of the World's Children 2003. New York: UNICEF; 2002.

8. Worrall, E., Basu, S. and Hanson, K. Is malaria a disease of poverty? A review of the literature. Trop. Med. Int. Health. 2005;10: 1047-1059.
9. Warhurst, D.C. and Williams, J.E. ACP Broadsheet no 148. July 1996. Laboratory diagnosis of malaria. J. Clin. Pathol. 1996; 49: 533-538.

10. Ochola, L.B., Vounatsou, P., Smith, T. et al. The reliability of diagnostic techniques in the diagnosis and management of malaria in the absence of a gold standard. Lancet. Infect. Dis. 2006; 6: 582-588.

11. Oloo, A.J., Ondijo, S.O., Genga, I.O. et al. Evaluation of the QBC method to detect malaria infections in field surveys. East Afr. Med. J. 1994; 71: 297-299.

12. Jelinek, T., Grobusch, M.P, Schwenke, S. et al. Sensitivity and specificity of dipstick tests for rapid diagnosis of malaria in nonimmune travelers. J. Clin. Microbiol. 1999; 37: 721-723.

13. Mills, C.D., Burgess, D.C., Taylor, H.J., et al. Evaluation of a rapid and inexpensive dipstick assay for the diagnosis of Plasmodium falciparum malaria. Bull. World Health Organ. 1999; 77: 553-559.

14. Rehlis, N. and Javor, P. Interpreteation of immunochromatographic tests with HRP-2 antigen in children under 5 years in an area of high risk of malaria transmission in Papua New Guinea. Wiad Parazytol. 2004; 50: 201-208.

15. Neumann, C.G., Bwibo, N.O., Murphy, S.P. et al. Animal source foods improve dietary quality, micronutrient status, growth and cognitive function in Kenyan school children: background, study design and baseline findings. J. Nutr. 2003; 133: (11 Suppl. 2) 3941S-3949S.

16. Beadle, C., Long, G.W., McElroy, P.D., et al. Diagnosis of malaria by detection of Plasmodium falciparum HRP-2 antigen with a rapid dipstick antigen-capture assay. Lancet. 1994; 343: 564-568.

17. Bell, D., Go R., Miguel, C., Walker, J.et al.A. Diagnosis of malaria in a remote area of the Philippines: comparison of techniques and their acceptance by health workers and the community. Bull. World Health Organ. 2001; 79: 933-941.

18. Cunha, M.L., Piovesan-Alves F. and Pang, L.W. Community-based program for malaria case management in the Brazilian Amazon. Am. J. Trop. Med. Hyg. 2001; 65: 872-876.

19. Banchongaksorn T., Prajakwong, S., Rooney, W. et al. Operational trial of ParasightTM-F (Dipstick) in the diagnosis of falciparum malaria at the primary health care level. Southeast Asian J. Trop. Med. Public Health. 1997; 23: 243-246.

20. Bell, D., Wongsrichanalai, C. and Barnwell, J.W. Ensuring quality and access for malaria diagnosis: how can it be achieved? Nat. Rev. Microbiol. 2006; 4: 682-695. 